\title{
Pyrosequencing 16S rRNA genes of bacteria associated with wild tiger mosquito Aedes albopictus: a pilot study
}

\author{
Guillaume Minard, Florence-Hélène Tran, Audrey Dubost, Van Tran-Van, Patrick Mavingui * and \\ Claire Valiente Moro*
}

Ecologie Microbienne, UMR CNRS 5557, USC INRA 1364, VetAgro Sup, FR41 BioEnvironment and Health, Université de Lyon 1, Villeurbanne, France

\author{
Edited by: \\ Muriel Vayssier-Taussat, INRA, \\ France \\ Reviewed by: \\ Fadi Bittar, Aix-Marseille Université, \\ URMITE, UM63, CNRS 7278, IRD \\ 198, Inserm 1095, France \\ Damien F. Meyer, CIRAD, France

\section{*Correspondence:} \\ Patrick Mavingui and Claire Valiente \\ Moro, Ecologie Microbienne, UMR \\ CNRS 5557, Université de Lyon 1, \\ Bâtiment Lwoff, 10, rue Raphaël \\ Dubois, 69100 Villeurbanne, France \\ e-mail: patrick.mavingui@ \\ univ-lyon 1.fr; \\ claire.valiente-moro@univ-lyon1.fr
}

The Asian tiger mosquito Aedes (Stegomya) albopictus is an invasive species that has spread across the world in the last two decades, showing a great capacity to adapt to contrasting climates and environments. While demonstrated in many insects, the contribution of bacterial symbionts in Aedes ecology is a challenging aspect that needs to be investigated. Also some bacterial species have already been identified in Ae. albopictus using classical methods, but a more accurate survey of mosquito-associated bacterial diversity is needed to decipher the potential biological functions of bacterial symbionts in mediating or constraining insect adaptation. We surveyed the bacteria associated with field populations of Ae. albopictus from Madagascar by pyrosequencing 16S rRNA gene amplicons. Different aspects of amplicon preparation and sequencing depth were tested to optimize the breadth of bacterial diversity identified. The results revealed that all mosquitoes collected from different sites have a bacterial microbiota dominated by a single taxon, Wolbachia pipientis, which accounted for about $99 \%$ of all 92,615 sequences obtained. As Ae. albopictus is known to harbor two Wolbachia strains (wAlbA and wAlbB), a quantitative PCR was used to estimate the relative densities, (i.e., the bacteria-to-host gene ratios) of each strains in individual mosquitoes. Relative densities were between $6.25 \times 10^{0.01}$ and $5.47 \times 10^{0.1}$ for wAlbA and between $2.03 \times 10^{0.1}$ and $1.4 \times 10^{1}$ for wAlbB. Apart from Wolbachia, a total of 31 bacterial taxa were identified at the genus level using different method variations. Diversity index values were low and probably underestimated the true diversity due to the high abundance of Wolbachia sequences vastly outnumbering sequences from other taxa. Further studies should implement alternative strategies to specifically discard from analysis any sequences from Wolbachia, the dominant endosymbiotic bacterium in Ae. albopictus from this area.

Keywords: high-throughput sequencing, 16S rDNA, bacterial diversity, Wolbachia, field-caught mosquitoes

\section{INTRODUCTION}

Invasive mosquitoes give cause for worldwide concern not only because of their potential ecological impact, but also they are vectors of a wide range of pathogens affecting both humans and animals (Medlock et al., 2012). In particular, the tiger mosquito Aedes albopictus has rapidly spread across the world from its native South-East Asia and is considered as one of the world's 100 most dangerous invasive species (Benedict et al., 2007). Ae. albopictus was found to be competent for more than 20 arboviruses (Gratz, 2004). Ae. albopictus was involved in the transmission of the Chikungunya and Dengue viruses worldwide, including to Europe (Bonilauri et al., 2008; Grandadam et al., 2011; Caron et al., 2012), and was also implicated as a vector of the parasitic nematode Dirofilaria (Cancrini et al., 2007).

In light of the recent theory of the holobiont unit, it is now necessary to enlarge our view of how commensal host-bacterial relationships function in insects (Feldhaar, 2011; Minard et al., 2013a). Insects establish numerous types of symbiotic associations ranging from parasitism to mutualism with their microbial communities (Toft and Andersson, 2010). Recent studies have highlighted how bacterial endosymbioses influence evolutionary and ecological processes through effects on insect host biology (Douglas, 2011). However, most examples demonstrate how symbiotic microbiota are essential to phytophagous insect functions such as host plant specialization (Tsuchida et al., 2004), reproduction (Simon et al., 2011), protection against natural enemies (Oliver et al., 2003), and tolerance to environmental stress (Brumin et al., 2011; Feldhaar, 2011). It is therefore necessary to assess how the bacterial microbiota contributes to the biology of hematophagous insects, particularly invasive mosquitoes.

Until now the composition of bacterial communities associated with Ae. albopictus has mainly been investigated using classical microbiological and molecular techniques. Various culture-dependent isolation methods were used to identify cultivable bacteria (Zouache et al., 2009; Chouaia et al., 2010; Minard et al., 2013a,b; Valiente Moro et al., 2013). In parallel, non-culture-based methods such as DGGE fingerprinting (Chouaia et al., 2010; Zouache et al., 2011) or taxonomic microarray hybridization (Zouache et al., 2012) were employed. Together these approaches have unraveled some of the bacterial diversity present in both laboratory-reared and field-caught Ae. albopictus 
mosquitoes. However, these relatively low-throughput techniques cannot provide comprehensive coverage of the microbial composition and diversity in mosquito hosts. The recent rapid development of high-throughput sequencing methods has made it possible to detect a deeper level of microbial diversity in animal hosts, it has been demonstrated for symbiotic microbiotas of insect guts (Shi et al., 2010; Fukatsu, 2012). To date very few studies have used Next-Generation Sequencing technologies to explore bacterial microbiota in mosquitoes. Osei-Poku et al. (2012) successfully used pyrosequencing of 16S rRNA genes to investigate the diversity of gut bacterial communities in field-caught mosquitoes and showed that bacterial composition varies greatly between populations. When pyrosequencing was used to determine the composition of the midgut microbiota of the malaria mosquito vector Anopheles gambiae, much richer information was obtained than from low-throughout methods (Wang et al., 2011; Boissière et al., 2012).

The goal of the present study was to survey the bacterial diversity associated with Ae. albopictus mosquitoes by pyrosequencing $16 \mathrm{~S}$ rDNA genes. The effect of varying different methodological parameters like those for amplicon preparation and sequencing depth on the bacterial diversity results was assessed.

\section{MATERIALS AND METHODS MOSOUITO COLLECTION}

The sampling area and mosquito capture procedure were approved by Madagascar National Parks. Mosquito specimens were collected from various natural breeding sites on the east coast of Madagascar (Toamasina) in the Antsinanana region $\left(18^{\circ} 8^{\prime} 59.64^{\prime \prime} \mathrm{S}\right)$ in December 2010. Butterfly nets were used to catch adult mosquitoes flying near the grass or around the capturers. Aedes albopictus specimens were identified using morphological characteristics and pictorial keys (Rueda, 2004) and then female and male mosquitoes were separated. Only non-blooded female mosquitoes were used for the analysis, as previous studies have demonstrated that blood meals have a dynamic effect on bacterial diversity (Wang et al., 2011; Boissière et al., 2012). Mosquitoes were stored in $70 \%$ ethanol at $-80^{\circ} \mathrm{C}$ until use.

\section{GENOMIC DNA EXTRACTION}

All DNA extractions were performed in a sterile environment under a laminar hood to avoid contamination. Mosquitoes were surface-disinfected with $70 \%$ ethanol and rinsed with sterile water, as previously described (Minard et al., 2013b). Each mosquito was individually crushed using a Bioblock Scientific MM 2000 mill (Retsch, France) in an Eppendorf tube with 5$\mathrm{mm}$ diameter inox beads and $200 \mu \mathrm{l}$ of extraction buffer $(2 \%$ hexadecyltrimethyl ammonium bromide, $1.4 \mathrm{M} \mathrm{NaCl}, 0.02 \mathrm{M}$ EDTA, $0.1 \mathrm{M}$ Tris $\mathrm{pH}$ 8.0, $0.2 \% \quad 2-\beta$-mercaptoethanol) and heated at $60^{\circ} \mathrm{C}$ for $2 \mathrm{~h}$. Lipids and proteins were extracted with phenol-chloroform-isoamyl alcohol $(25: 24: 1 ; \mathrm{v} / \mathrm{v} / \mathrm{v})$ and then chloroform:isoamyl alcohol $(24: 1 ; \mathrm{v} / \mathrm{v})$. DNA was precipitated with isopropyl alcohol and centrifugation at $16,100 \mathrm{~g}$ for $30 \mathrm{~min}$ at $4^{\circ} \mathrm{C}$. DNA pellets were washed with cold ethanol, airdried, and resuspended in $20 \mu \mathrm{l}$ TE buffer $(2 \mathrm{mM}$ Tris, $1 \mathrm{mM}$ EDTA).

\section{PRIMERS AND PCR AMPLIFICATION}

Hypervariable rrs gene V5-V6 regions of about $280 \mathrm{bp}$ were amplified with 784F 5'-AGGATTAGATACCCTGGTA-3' and 1061R 5'-CRRCACGAGCTGACGAC-3' primers (Andersson et al., 2008). These primers were selected in silico with the RDP Probe Match tool according to the following criteria (Cole et al., 2009): hybridization with $94 \%$ of sequences belonging to the RDP Bacteria domain database with good quality and $\geq 1200 \mathrm{bp}$ long with 2 mismatches allowed in primer sequences. In addition, Basic Local Alignment Search Tool (BLAST) was used to check that the chosen primers did not match Ae. albopictus $18 \mathrm{~S}$ and mitochondrial 16S rDNA gene sequences. PCR amplification was performed using $1.75 \mathrm{U}$ of Expand High Fidelity Enzyme Mix (Roche, Switzerland) with $1 \times$ Expand High Fidelity Buffer (Roche, Switzerland), $0.06 \mathrm{mg} \mathrm{mL}^{-1}$ of T4 gene 32 protein (Roche, France), $0.06 \mathrm{mg} \mathrm{mL}^{-1}$ of bovine serum albumin (New England Biolabs, France), $40 \mu \mathrm{M}$ of dNTP mix, $200 \mathrm{nM}$ of each primer (Invitrogen, France) and $30 \mathrm{ng}$ of template DNA. PCR products were purified with QiaAmp purification kit (Qiagen, France) following the manufacturer's recommendations.

\section{PREPARATION OF SAMPLES FOR 454 PYROSEOUENCING AND BIOINFORMATIC PROCESSING}

Amplicon tagging and pyrosequencing were performed by a commercial laboratory (DNAvision, Belgium) using the Roche 454 FLX Titanium platform (Roche, Switzerland). Various modalities were tested for in-depth exploration of bacterial diversity (Figure 1) including: (i) constituting a pool of DNA then performing PCR amplification vs. pooling PCR of several amplicons generated from different individual samples; (ii) sequencing a single amplicon vs. a pool of amplicons; and (iii) evaluating the depth of sequencing (number of reads) necessary for the most complete examination of the bacterial diversity. A total of eight rrs V5-V6 amplicon libraries were sequenced at two different ranges of about 3200 reads per sample (S1, S2, S3, S4, S5) and 16,000 reads per sample $\left(\mathrm{S}^{\prime}, \mathrm{P} 1, \mathrm{P} 2\right)$ (Figure 1). Data were analyzed by first trimming sequence quality with different cutoffs using standard filtering tools in the Mothur package (Schloss et al., 2009). Two errors on primer sequence and one error on barcode sequence were allowed. Sequences shorter than $250 \mathrm{bp}$ were discarded and chimeric sequences were removed using UCHIME and manual analysis (Edgar et al., 2011). Sequences were grouped into operational taxonomic units (OTUs) by clustering at $97 \%$ similarity. Singleton reads assigned to OTUs were discarded from the analysis if they were not found in at least two different samples as singletons are likely to be sequencing errors which can lead to an overestimation of diversity. Rarefaction curves were built to estimate sample coverage. Richness, $\alpha$ diversity and $\beta$ diversity were calculated to compare samples with respectively richness estimators (Chao, Jackknife, Abundance Coverage Estimator), diversity indices (Simpson, Shannon) and Bray-Curtis dissimilarity. Bray-Curtis dissimilarity values were used to construct a neighbor joining tree with the BIONJ algorithm (Gascuel, 1997) using R software (R Development Core Team, 2009). OTU consensus sequences were screened against the SILVA database (release 111) with a 5\% dissimilarity cut-off and an $e$-value of $10^{-12}$. Taxonomic assignment of sequence clusters 


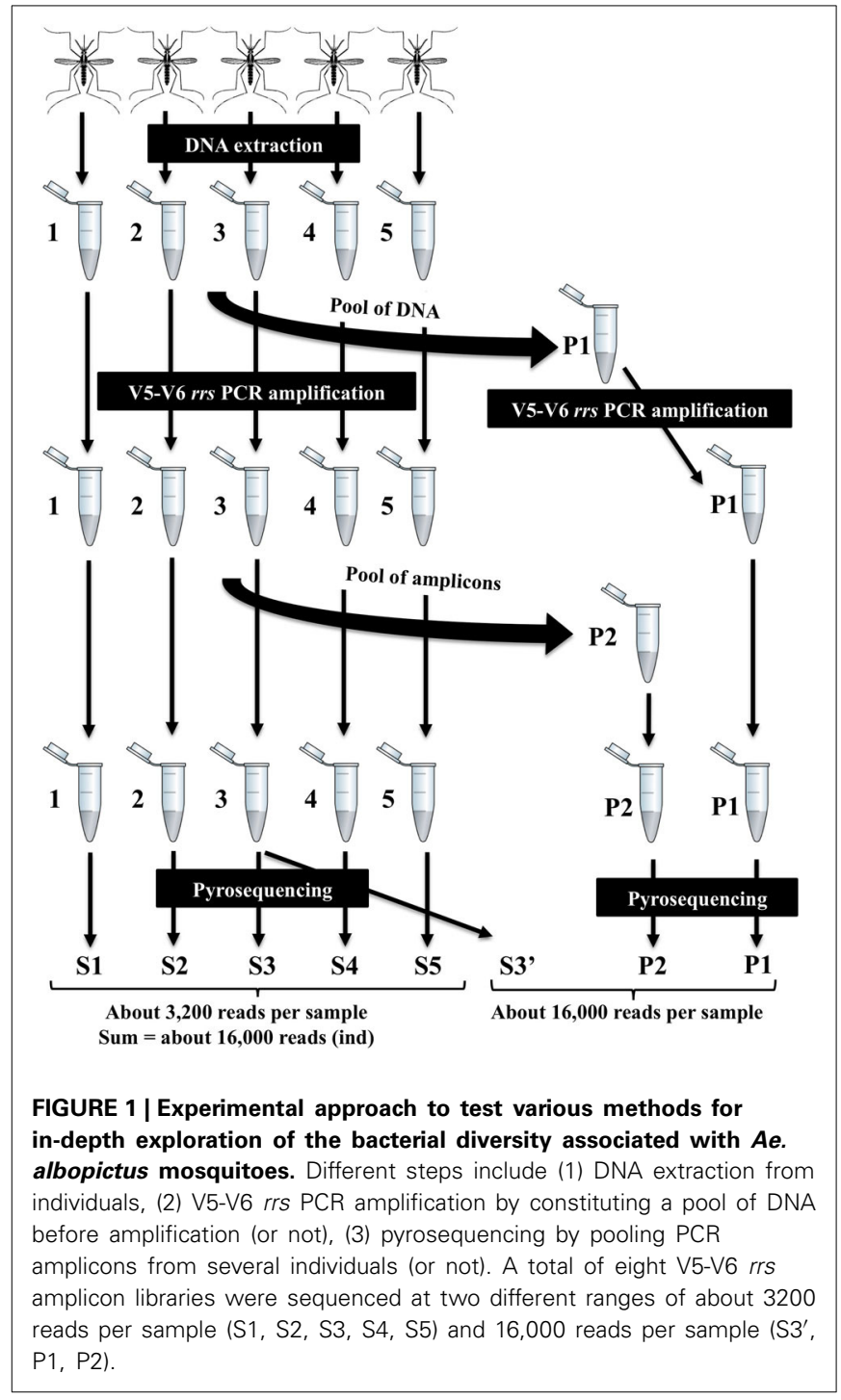

was performed with local BLASTALL searches. A new sample, named ind, was added to the analysis. This sample was generated by pooling all the reads from each individual sample ( $\mathrm{S} 1$ to S5) (Figure 1). All sff files were deposited at EMBL European Nucleotide Archive (www.ebi.ac.uk/ena/) under project accession number PRJEB4976.

\section{QUANTIFICATION OF WOLBACHIA}

The relative density of Wolbachia pipientis was measured by real-time quantitative PCR specifically targeting the wsp genes for each of the Wolbachia strains wAlbA and wAlbB (Zouache et al., 2011). Amplification using the LightCycler LC480 apparatus (Roche) was performed using $5 \mathrm{ng}$ of DNA from a single female mosquito (10 females in total), as previously described (Zouache et al., 2011). Standard curves were constructed using a dilution series $\left(10^{1}-10^{8}\right.$ molecules) of the pQuantAlb plasmid containing wsp and actin fragments (Tortosa et al., 2008). For each gene, three technical replicates were processed per DNA sample.

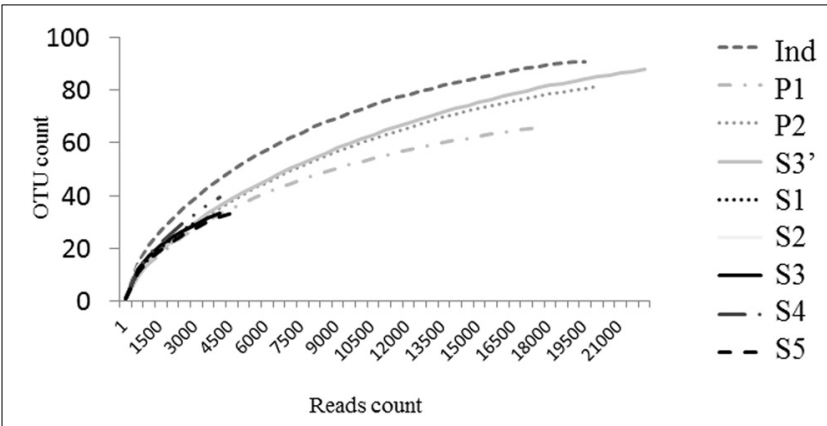

FIGURE 2 | Rarefaction analysis of bacterial microbiota discovery. Operational taxonomic units (OTUs) were grouped with a $97 \%$ similarity. Rarefaction curves represent the number of new OTUs discovered by sampling without being recaught.

\section{RESULTS}

\section{BACTERIAL SPECIES RICHNESS AND DIVERSITY IN MOSOUITOES}

The V5-V6 regions of bacterial rrs genes amplified from female Ae. albopictus adults were sequenced by high-throughput pyrosequencing. From the total of 92,615 reads obtained, approximately 79,895 were judged to be of good quality. For samples from individual mosquitoes (S1, S2, S3, S4, S5), read numbers varied from 1583 (S2) to 5048 (S5). For P1, P2, Ind, and S3', read numbers were $17,584,20,447,19,132$, and 22,199, respectively. Sequences clustered at $97 \%$ similarity into 417 OTUs. The coverage of the sequencing was determined by using rarefaction analysis. The rarefaction curves of samples from single mosquitoes did not reach saturation (Figure 2). On the contrary, saturation was almost attained for the ind pool of individual sample sequences (S1-S5), P1 sequences obtained from amplicons generated from a pool of DNA and P2 sequences obtained from a pool of amplicons (i.e., after amplification of a single sample). The comparison of rarefaction curves between S3 (4276 reads) and S3' (22,296 reads) clearly indicated that for a single mosquito individual a minimal sequence number close to 16,000 reads is sufficient for a good estimation of sample richness. Indeed, diversity indices Chao1, ACE1, Jackknife for S3 are about half of the respective S3' values (Table 1). However, the Simpson and Shannon diversity indices of S3 and S3' were similar. In addition, analyses of Bray-Curtis distances showed a low level of dissimilarity $(B C=0.03939)$ between S3 and S3'. Other dissimilarity scores revealed that overall the $\mathrm{P} 2$ and ind pools $(B C=0.059085)$ are more similar to each other than to P1 (Figure 3).

\section{WOLBACHIA SEQUENCES ARE THE PREDOMINANT 16S rDNA AMPLICONS}

A total of 417 OTUs were assigned to 4 phyla and 32 genera (Figure 4). Sequences from Proteobacteria are the predominant sequences found in all samples with all the experimental modalities tested ( $>99 \%$ of reads). About 96 to $99 \%$ of reads belonged to the Wolbachia genus and this largely explains the low diversity values obtained in each condition (Table 1). To confirm sequences assignment to Wolbachia, a local alignment of the two most abundant OTUs (Wolb G1 and Wolb G2) was performed against the non-redundant NCBI database to allow the 
Table 1 | Richness and diversity index.

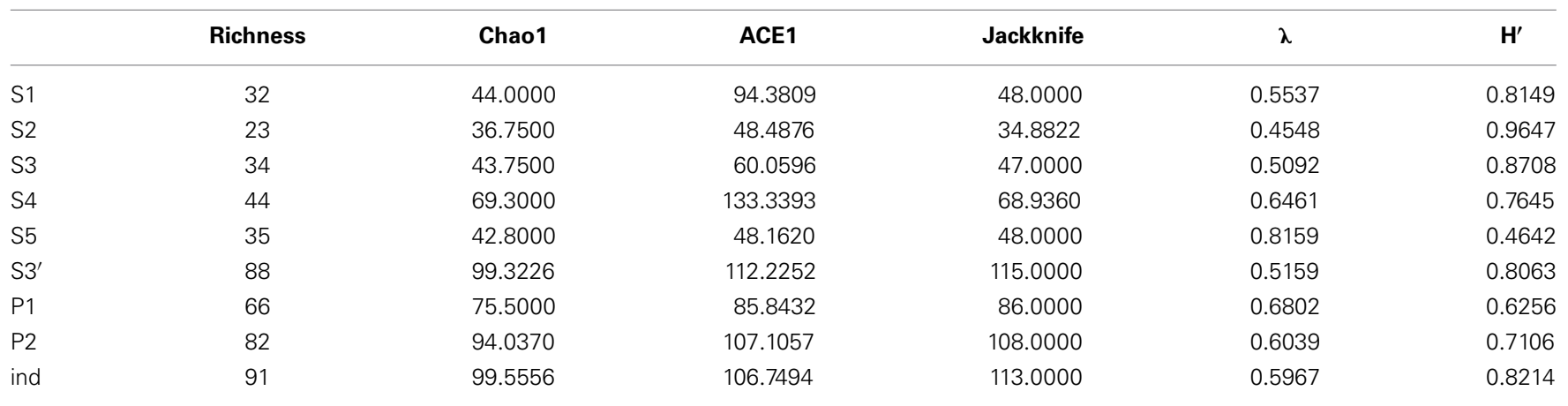

Indexes were calculated for each sample with 3\% distant OTUs.

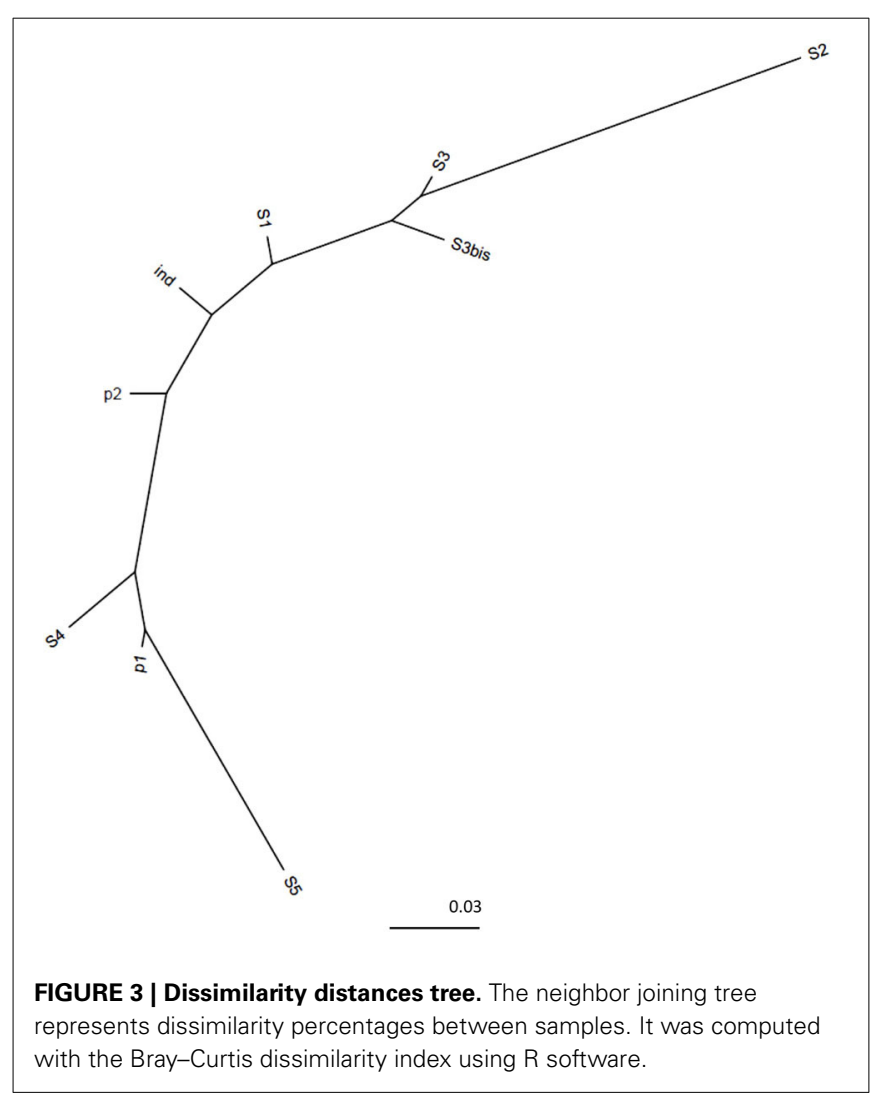

selection of sequences from the most closely related taxa. The latter sequences were then aligned with Wolb G1 and Wolb G2 sequences and with 15 Wolbachia sequences retrieved from GenBank. This alignment highlighted the presence of a specific 25-bp sequence in the Wolbachia rrs gene (Figure S1). The alignment of this region with public databases was also performed using the SeqMatch tool (http://rdp.cme.msu.edu). The sequence showed $100 \%$ similarity and query coverage with other Wolbachia sequences but only $57 \%$ query coverage and $100 \%$ similarity with the nearest neighbor sequence from different taxa including Candidatus mikurensis, Ehrlichia ruminantium, and Anaplasma platys. This confirmed that the 25-bp sequence was specific to Wolbachia.
The numbers of Wolbachia $w$ AlbA and $w$ AlbB strains present in 10 mosquito individuals were quantified by clade-specific quantitative PCR of $w s p$, a gene encoding for Wolbachia surface protein, normalized to actin, a mosquito housekeeping gene. For each strain a relatively high gene copy number was found in all individuals tested (Figure 5). The wsplactin ratios were between $6.25 \times 10^{0.01}$ and $5.47 \times 10^{0.1}$ for $w \mathrm{AlbA}$ and between $2.03 \times 10^{0.1}$ and $1.4 \times 10^{1}$ for $w$ AlbB. Significant bacterial density differences were found between individual mosquitoes for both $w$ AlbA ( $p$-value $=8.499 \times 10^{-15}$, Kruskall-Wallis test $)$ and $w$ AlbB $\left(p\right.$-value $=3.616 \times 10^{-14}$, Kruskall-Wallis $)$.

\section{TAXONOMIC ANALYSIS AND RELATIVE ABUNDANCE OF OTHER BACTERIAL TAXA}

Apart from Wolbachia, a total of 31 bacterial taxa were identified at the genus level from less than $1 \%$ of all the sequences (Figure 4). Firmicutes bacteria were found to be the dominant group (67\%), followed by Actinobacteria (4\%), Bacteroidetes $(3 \%)$, and Proteobacteria (2\%). In terms of OTU richness and genus content, ind and $\mathrm{P} 2$ were the most diverse with 22 and 19 genera identified, respectively (Tables 1, 2). Sequences corresponding to Blautia, a Firmicutes genus, were found in all the datasets from individual and pooled samples, ranging from $3.2 \%$ (for P1) to $89.2 \%$ (for S4) of sequences (Table 2). Moreover, 88 sequences from a single OTU were assigned to Lachnospiaceae family with no similarity at genus level. Overall, the proportion of sequences affiliated to known or unclassified bacterial genera varied among samples.

\section{DISCUSSION}

The application of next-generation sequencing techniques to insect microbiota research makes it possible to gain a deeper knowledge of its microbial diversity. The goal of the present study was to assess the diversity of bacteria associated with the Asian tiger mosquito using high-throughput sequencing. Previous studies of bacterial diversity in various arthropods using a similar approach gave rise to different methodological parameters (Khöler et al., 2012; Moran et al., 2012; Osei-Poku et al., 2012; Ridley et al., 2012), so we chose to carry out a pilot study using pyrosequencing on a hypervariable region of $16 \mathrm{~S}$ rRNA genes. Various experimental modalities were tested by varying the sample preparation (processing DNA samples from 
Table 2 | Relative abundance of different genera without Wolbachia.

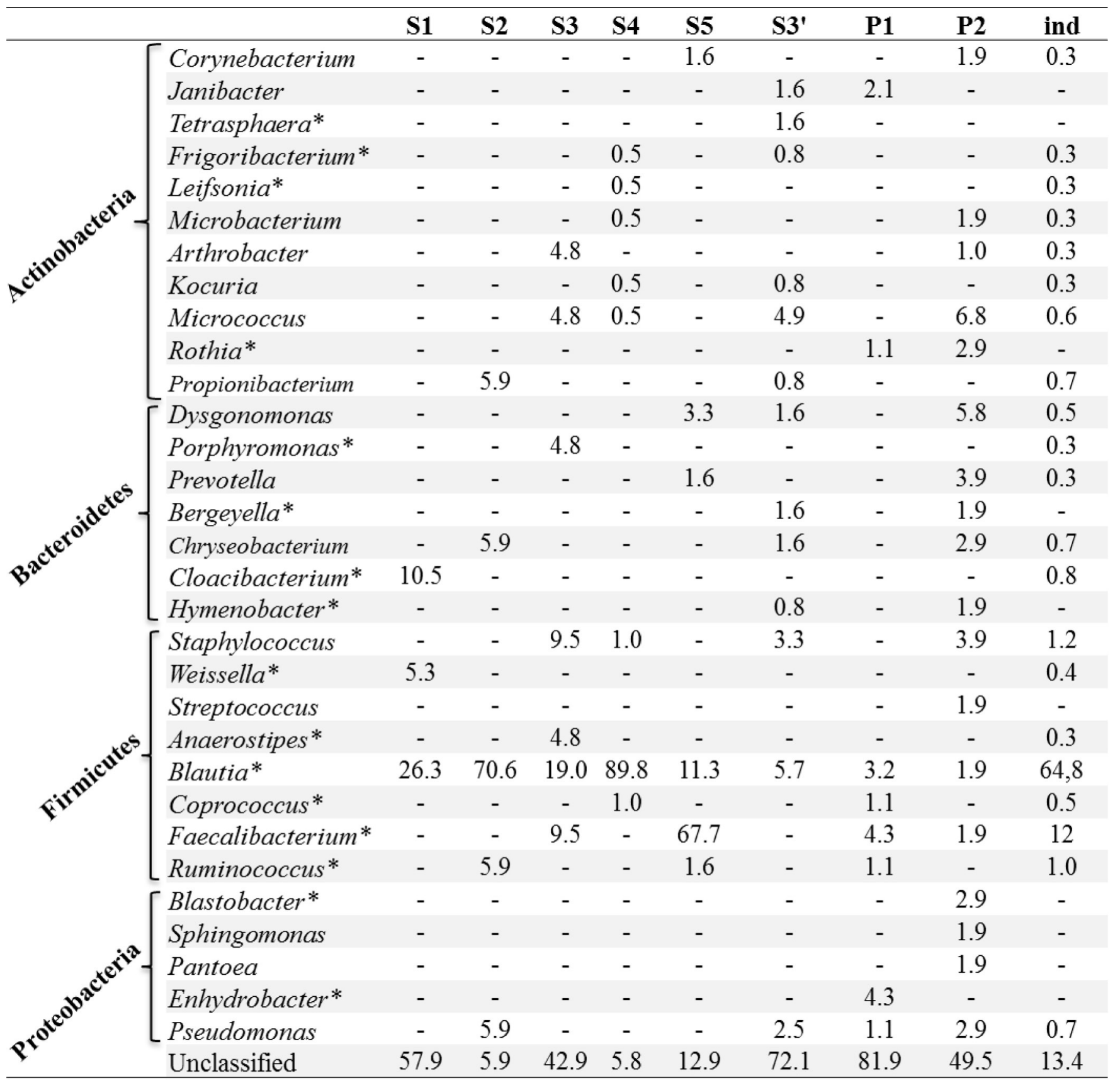

The asterisks $\left(^{*}\right)$ represent newly identified genera in mosquitoes according to Minard et al., $2013 a, b$.

individuals or mixing several $16 \mathrm{~S}$ amplicons either before or after PCR amplification) and depth of sequencing (number of reads). The primers chosen targeted the V5-V6 hypervariable region of $16 \mathrm{~S}$ rRNA which allows a large number of bacterial taxa to be detected from sequences available in databases $(94 \%$ of RDP database) and takes into account other limiting factors such as amplicon size and a lack of hybridization with host DNA.

The results obtained show first that a depth of at least 16,000 reads was necessary to accurately reach the saturation of richness discovery curves. Indeed, the observed richness was made to approach the estimated richness by increasing the depth of sequencing as demonstrated by comparing S3 (4276 reads) and S3' (22,296 reads). In addition, as these two datasets originated from the same amplicon sample sequenced independently, the low Bray-Curtis dissimilarity levels between them reflects experimental reproducibility. Another factor evaluated was whether the pooling of samples would prove a cost-effective way to compare a huge number of samples. The drawback is that pooling may hide variability 

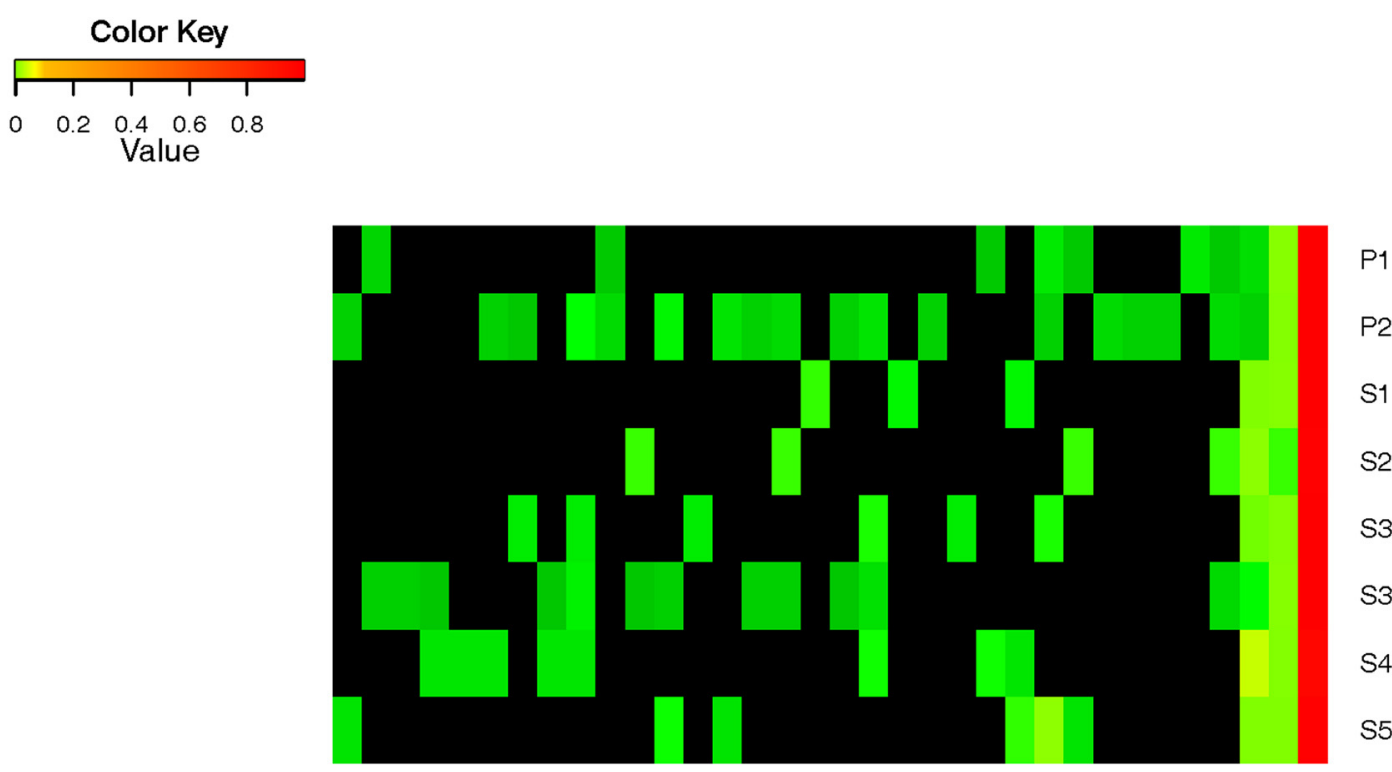

P1

P2

S1

S2

S3

$\frac{\infty}{d}$

S3'

S4

55

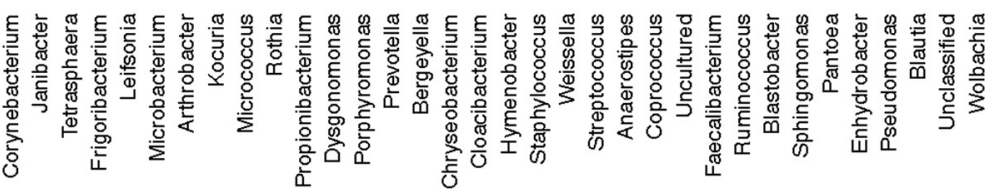

Genera

FIGURE 4 | Relative abundance of bacterial genera in Aedes albopictus. Heatmap represent the proportions of OTUs at the genus level.

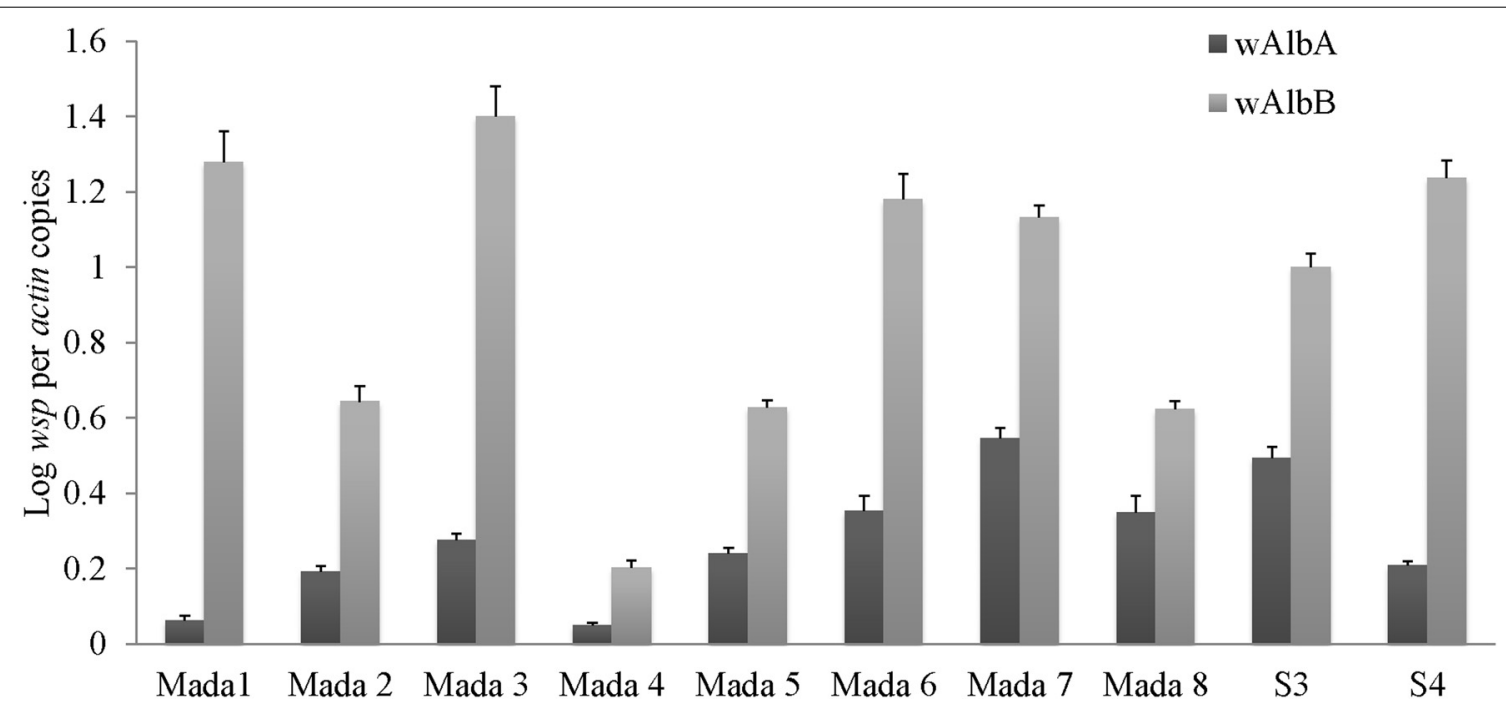

FIGURE 5 | Wolbachia titers in Aedes albopictus individuals. The ratio of Wolbachia wsp gene to Aedes albopictus actin gene copies is reported for wAlbA and wAlbB strains in S3 and S4 samples and for 8 other samples from the same site (Mada1 to Mada 8).

in the bacterial composition among samples. Here, we showed that it is preferable to pool amplicons rather than DNA before amplification as the latter method clearly failed to detect rare sequences.
In this study, pyrosequencing amplicons from Ae. albopictus bacterial microbiota revealed a lower Simpson diversity index than previously obtained with fingerprint methods (Zouache et al., 2011). However, using 454 pyrosequencing of $16 \mathrm{~S}$ rRNA 
fragments, Osei-Poku et al. (2012) found that 23 genera occurred at a frequency of more than $1 \%$ in the guts of at least one individual of different mosquito species. They also reported very low bacterial diversity within individuals, with a single OTU typically making up two-thirds of all the bacteria in all field-caught individuals from seven species studied, which is similar to our results. Interestingly, the results presented here reveal the highest genus richness that we have yet found in Ae. albopictus using various methods (Zouache et al., 2011, 2012; Valiente Moro et al., 2013). The results show that high throughput sequencing is an efficient way to deeply investigate microbiota composition in mosquitoes.

We conclude that Wolbachia was the principle component of Ae. albopictus microbiota in different individuals as surprisingly about $99 \%$ of reads were Wolbachia sequences independent of the experimental method used. The relative densities of Wolbachia $w \mathrm{AlbA}$ and $w \mathrm{AlbB}$ in both field-caught and lab-reared Ae. albopictus have been measured by quantitative PCR and the values we report here are similar to those previously found in fieldcaught populations (Tortosa et al., 2010; Zouache et al., 2011, 2012). All mosquito samples analyzed here were positive for both strains. Possibly the abundance of Wolbachia and strain bi-infection confers a fitness advantage like that described in Wolbachia-superinfected mosquitoes (Dobson et al., 2002, 2004). This selective pressure that acts on hatching rates and lifespan in laboratory conditions could be a reason for the predominance of Wolbachia in field populations mitigating the metabolic cost of symbiosis. Cytoplasmic incompatibility induced by Wolbachia is also responsible for a high prevalence of superinfected females (Kittayapong et al., 2002; Dobson et al., 2004; Zouache et al., 2011).

Sequences affiliated to Blautia were the second most abundant after those from Wolbachia. This is the first report of the presence of this genus in Ae. albopictus and even in mosquitoes. Blautia are anaerobic acetogenic members of Firmicutes and some have been isolated from mammal guts (Bernalier et al., 1996; Gagen et al., 2010). In addition to Blautia, we discovered other anaerobic bacteria in Ae. albopictus, including Faecalibacterium, Ruminococcus, and Coprococcus. Some of these are thought to be involved in the metabolism of organic compounds such as acetic acid in the human gut (Tsai et al., 1976; Wrzosek et al., 2013). Of the 32 bacterial genera identified overall, 9 had been previously found in Ae. albopictus and 8 others had already been described in other mosquito species using low throughput molecular techniques (reviewed in Minard et al., 2013a). Among these bacteria, the genus Pantoea is one of the most prevalent bacterial cultivable groups identified in field-caught Ae. albopictus from Madagascar (Valiente Moro et al., 2013). The confirmation of the presence of these bacterial taxa in Ae. albopictus highlights the urgency of characterizing the metabolic aspects of these host-microbe interactions in relation to habitat specialization. Stinkingly, some human opportunistic pathogens belong to genera that were identified in mosquitoes such as Pseudomonas, Staphylococcus, Chryseobacterium, and Dysgonomonas (Hironaga et al., 2008; Asaad et al., 2013; Dettman et al., 2013; Ozcan et al., 2013; Coates et al., 2014). Linked to the contact between mosquito proboscis and human circulation during females' blood feeding, these observations argue the possibility of a mosquito-mediated bacterial transmission. Finally, a number of sequences were not assigned to any bacterial family but sequencing longer amplicons might improve their identification rate.

\section{CONCLUSION}

Here we developed a high-throughput sequencing protocol to phylotype bacterial communities associated with Ae. albopictus mosquitoes. To our knowledge, this is the first time that such an approach has been used to explore Ae. albopictus microbiota. Although the results reveal the most complete picture of the bacterial microbiota in any given insect, they also show the limits of the high-throughput sequencing approach when there is a dominant endosymbiotic species. Alternative strategies such as the use of blocking primers (Vestheim et al., 2011) will be implemented to specifically discard sequences of the particularly abundant Wolbachia endosymbiont. The NGS approach developed in this study will be helpful to perform a large scale analysis of mosquitoassociated microbiota from different geographic locations and would allow exploring the role of bacteria in invasive capacities of mosquitoes.

\section{AUTHOR CONTRIBUTIONS}

Guillaume Minard, Claire Valiente Moro, and Patrick Mavingui conceived and designed the work. Guillaume Minard, FlorenceHélène Tran, and Van Tran-Van performed the experiments. Guillaume Minard and Audrey Dubost managed the data mining. Guillaume Minard, Claire Valiente Moro, and Patrick Mavingui analyzed and interpreted the data, and drafted the manuscript and the final version was approved by all the authors.

\section{ACKNOWLEDGMENTS}

We thank Maxime Bruto for his help in phylogeny analysis. Funding for this project was provided by grants from EC2CO CNRS and CMIRA Région Rhône-Alpes. This research was also partially funded by the ERA-NET BiodivERsA, with the national funders FRB France, FWF Austria, and DFG Germany, part of the 2012-2013 BiodivERsA call for research proposals, and was carried out within the frameworks of GDRIs "Biodiversité et Développement Durable à Madagascar" and "Biodiversity and Infectious Diseases in Southeast Asia."

\section{SUPPLEMENTARY MATERIAL}

The Supplementary Material for this article can be found online at: http://www.frontiersin.org/journal/10.3389/fcimb. 2014.00059/abstract

Figure S1 | Alignment of Wolbachia reads. Wolbachia reads Wolb G1 and Wolb G2 were aligned with sequences from most closely related taxa (based on V5-V6 rrs sequences) and other Wolbachia sequences retrieved from GenBank, using Jalview software. A 25-bp Wolbachia specific region is highlighted.

\section{REFERENCES}

Andersson, A. F., Lindberg, M., Jakobsson, H., Nyrén, P., and Engstrand, L. (2008). Comparative analysis of human gut microbiota by barcoded pyrosequencing. PLoS ONE 3:e2836. doi: 10.1371/journal.pone.0002836

Asaad, M. A., Al-Ayed, M. S., and Quresh, M. A. (2013). Emergence of unusual nonfermenting gram-negative nosocomial pathogens in a Saudi hospital. Jpn. J. Infect. Dis. 66, 507-511. doi: 10.7883/yoken.66.507 
Benedict, M. Q., Levine, R. S., Hawley, W. A., and Lounibos, L. P. (2007). Spread of the tiger: global risk of invasion by the mosquito Aedes albopictus. Vector Borne Zoonotic Dis. 7, 76-85. doi: 10.1089/vbz.2006.0562

Bernalier, A., Willems, A., Leclerc, M., Rochet, V., and Collins, M. D. (1996). Ruminococcus hydrogenotrophicus sp. nov., a new $\mathrm{H} 2 / \mathrm{CO} 2$-utilizing acetogenic bacterium isolated from human feces. Arch. Microbiol. 166, 176-183. doi: 10.1007/s002030050373

Boissière, A., Tchioffo, M. T., Bachar, D., Abate, L., Marie, A., Nsango, S. E., et al. (2012). Midgut microbiota of the malaria mosquito vector Anopheles gambiae and interactions with Plasmodium falciparum infection. PLOS Pathog. 8:e1002742. doi: 10.1371/journal.ppat.1002742

Bonilauri, P., Bellini, R., Calzolari, M., Angelini, R., Venturi, L., Fallacara, F., et al. (2008). Chikungunya virus in Aedes albopictus, Italy. Emerg. Infect. Dis. 14, 852-854. doi: 10.3201/eid1405.071144

Brumin, M., Kontsedalov, S., and Ghanim, M. (2011). Rickettsia influences thermotolerance in the whitefly Bemisia tabaci B biotype. Insect Sci. 18, 57-56. doi: $10.1111 /$ j.1744-7917.2010.01396.x

Cancrini, G., Scaramozzino, P., Gabrielli, S., Di Paolo, M., Toma, L., and Romi, R. (2007). Aedes albopictus and Culex pipiens implicated as natural vectors of Dirofilaria repens in central Italy. J. Med. Entomol. 44, 1064-1066. doi: 10.1603/0022-2585(2007)44[1064:AAACPI]2.0.CO;2

Caron, M., Paupy, C., Grard, G., Becquart, P., Mombo, I., Nso, B. B., et al. (2012). Recent introduction and rapid dissemination of Chikungunya virus and Dengue virus serotype 2 associated with human and mosquito coinfections in Gabon, central Africa. Clin. Infect. Dis. 55, e45-e53. doi: 10.1093/cid/cis530

Chouaia, B., Rossi, P., Montagna, M., Ricci, I., Crotti, E., Damiani, C., et al. (2010). Molecular evidence for multiple infections as revealed by typing of Asaia bacterial symbionts of four mosquito species. Appl. Environ. Microbiol. 76, 7444-7450. doi: 10.1128/AEM.01747-10

Coates, R., Moran, J., and Horsbugh, M. J. (2014). Staphylococci: colonizers and pathogens of human skin. Future Microbiol. 9, 75-91. doi: 10.2217/fmb.13.145

Cole, J. R., Wang, Q., Cardenas, E., Fish, J., Chai, B., Farris, R. J., et al. (2009). The Ribosomal Database Project: improved alignments and new tools for rRNA analysis. Nucleic Acids Res. 37, D141-D145. doi: 10.1093/nar/gkn879

Dettman, J. R., Rodrigue, N., Aaron, S. D., and Kassen, R. (2013). Evolutionary genomics of epidemic strains of Pseudomonas aeruginosa. Proc. Natl. Acad. Sci. U.S.A. 110, 21065-21070. doi: 10.1073/pnas.1307862110

Dobson, S. L., Marsland, E. J., and Rattanadechakul, W. (2002). Mutualistic Wolbachia infection in Aedes albopictus: accelerating cytoplasmic drive. Genetics 160, 1087-1094.

Dobson, S. L., Rattanadechakul, W., and Marsland, E. J. (2004). Fitness advantage and cytoplasmic incompatibility in Wolbachia single- and superinfected Aedes albopictus. Heredity 93, 135-142. doi: 10.1038/sj.hdy.6800458

Douglas, A. E. (2011). Lessons from studying insect symbioses. Cell Host Microbe 10, 359-367. doi: 10.1016/j.chom.2011.09.001

Edgar, R. C., Haas, B. J., Clemente, J. C., Quince, C., and Knight, R. (2011). UCHIME improves sensitivity and speed of chimera detection. Bioinformatics 27, 2194-2200. doi: 10.1093/bioinformatics/btr381

Feldhaar, H. (2011). Bacterial symbionts as mediators of ecologically important traits of insect hosts. Ecol. Entomol. 36, 533-543. doi: 10.1111/j.13652311.2011.01318.x

Fukatsu, T. (2012). Next-generation sequencing sheds light on intricate regulation of insect gut microbiota. Mol. Ecol. 21, 5908-5910. doi: 10.1111/mec. 12090

Gagen, E. J., Denman, S. E., Padmanabha, J., Zadbuke, S., Al Jassim, R., Morrison, M., et al. (2010). Functional gene analysis suggests different acetogen populations in the bovine rumen and tammar wallaby forestomach. Appl. Environ. Microbiol. 76, 7785-7795. doi: 10.1128/AEM.01679-10

Gascuel, O. (1997). BIONJ: an improved version of the NJ algorithm based on a simple model of sequence data. Mol. Biol. Evol. 14, 685-695. doi: 10.1093/oxfordjournals.molbev.a025808

Grandadam, M., Caro, V., Plumet, S., Thiberge, J. M., Souarès, Y., Failloux, A. B., et al. (2011). Chikungunya virus, southeastern France. Emerg. Infect. Dis. 17, 910-913. doi: 10.3201/eid1705.101873

Gratz, N. G. (2004). Critical review of the vector status of Aedes albopictus. Med. Vet. Entomol. 18, 215-227. doi: 10.1111/j.0269-283X.2004.00513.X

Hironaga, M., Yamane, K., Inaba, M., Haga, Y., and Arakawa, Y. (2008). Characterization and antimicrobial succeptibility of Dysgonomonas capnocytophagoides isolated from human blood sampe. Jpn. J. Infect. Dis. 61, 212-213.
Kittayapong, P., Baisley, K. J., Sharpe, R. G., Baimai, V., and O’Neill, S. L. (2002). Maternal transmission efficiency of Wolbachia superinfections in Aedes albopictus populations in Thailand. Am. J. Trop. Med. Hyg. 66, 103-107.

Khöler, T., Dietrich, C., Scheffrahn, R. H., and Brune, A. (2012). High-resolution analysis of gut environment and bacterial microbiota reveals functional compartmentation of the gut in wood-feeding higher termites (Nasutitermes spp.). Appl. Environ. Microbiol. 78, 4691-4701. doi: 10.1128/AEM.00683-12

Medlock, J. M., Hansford, K. M., Schaffner, F., Versteirt, V., Hendrickx, G., Zeller, H., et al. (2012). A review of the invasive mosquitoes in Europe: ecology, public health risks, and control options. Vector Borne Zoonotic Dis. 12, 435-447. doi: $10.1089 / \mathrm{vbz} .2011 .0814$

Minard, G., Mavingui, P., and Moro, C. V. (2013a). Diversity and function of bacterial microbiota in the mosquito holobiont. Parasit. Vectors 6, 146. doi: 10.1186/1756-3305-6-146

Minard, G., Tran, F. H., Raharimalala, F. N., Hellard, E., Ravelonandro, P., Mavingui, P., et al. (2013b). Prevalence, genomic and metabolic profiles of Acinetobacter and Asaia associated with field-caught Aedes albopictus from Madagascar. FEMS Microbiol. Ecol. 83, 63-73. doi: 10.1111/j.15746941.2012.01455.x

Moran, N. A., Hansen, A. K., Powell, J. E., and Sabree, Z. L. (2012). Distinctive gut microbiota of honey bees assessed using deep sampling from individual worker bees. PLOS ONE 7:e36393. doi: 10.1371/journal.pone.0036393

Oliver, K. M., Russell, J. A., Moran, N. A., and Hunter, M. S. (2003). Facultative bacterial symbionts in aphids confer resistance to parasitic wasps. Proc. Natl. Acad. Sci. U.S.A. 100, 1803-1807. doi: 10.1073/pnas.0335320100

Osei-Poku, J., Mbogo, C. M., Palmer, W. J., and Jiggins, F. M. (2012). Deep sequencing reveals extensive variation in the gut microbiota of wild mosquitoes from Kenya. Mol. Ecol. 21, 5138-5150. doi: 10.1111/j.1365-294X.2012.05759.x

Ozcan, N., Tuba, D., Tekin, A., Kelekci, S., Can, S., Ezin, O., et al. (2013). Is Chryseobacterium indologenes a shunt-lover bacterium? A case report and review of the literature. Infez. Med. 4, 312-316.

R Development Core Team. (2009). R: A Language and Environment for Statistical Computing. Vienna: R Foundation for Statistical Computing. Available online at: http://www.R-project.org

Ridley, E. V., Wong, A. C., Westmiller, S., and Douglas, A. E. (2012). Impact of the resident microbiota on the nutritional phenotype of Drosophila melanogaster. PLoS ONE 7:e36765. doi: 10.1371/journal.pone.0036765

Rueda, L. M. (2004). Pictorial keys for the identification of mosquitoes (Diptera: Culicidae) associated with Dengue Virus Transmission. Zootaxa 589, 33-41.

Schloss, P. D., Westcott, S. L., Ryabin, T., Hall, J. R., Hartmann, M., Hollister, E. B., et al. (2009). Introducing mothur: open-source, platform-independent, community-supported software for describing and comparing microbial communities. Appl. Environ. Microbiol. 75, 7537-7541. doi: 10.1128/AEM. 01541-09

Shi, W., Syrenne, R., Sun, J. Z., and Yuan J. S. (2010). Molecular approaches to study the insect gut symbiotic microbiota at the "omics" age. Insect Sci. 17, 199-219. doi: 10.1111/j.1744-7917.2010.01340.x

Simon, J. C., Boutin, S., Tsuchida, T., Koga, R., Le Gallic, J. F., Frantz, A., et al. (2011). Facultative symbiont infections affect aphid reproduction. PLoS ONE 6:e21831. doi: 10.1371/journal.pone.0021831

Toft, C., and Andersson, S. G. (2010). Evolutionary microbial genomics: insights into bacterial host adaptation. Nat. Rev. Genetics. 11, 465-475. doi: $10.1038 / \mathrm{nrg} 2798$

Tortosa, P., Charlat, S., Labbé, P., Dehecq, J. S., Barré, H., and Weill, M. (2010). Wolbachia age-sex-specific density in Aedes albopictus: a host evolutionary response to cytoplasmic incompatibility? PLoS ONE 5:e9700. doi: 10.1371/journal.pone. 0009700

Tortosa, P., Courtiol, A., Moutailler, S., Failloux, A. B., and Weill, M. (2008). Chikungunya-Wolbachia interplay in Aedes albopictus. Insect. Mol. Biol. 17, 677-684. doi: 10.1111/j.1365-2583.2008.00842.x

Tsai, C. G., Gates, D. M., Indledew, W. M., and Jones, G. A. (1976). Products of anaerobic phloroglucinol degradation by Coprococcus sp. Pe15. Can. J. Microbiol. 22, 159-164. doi: 10.1139/m76-022

Tsuchida, T., Koga, R., and Fukatsu, T. (2004). Host plant specialization governed by facultative symbiont. Science 303, 1989. doi: 10.1126/science. 1094611

Valiente Moro, C., Tran, F. H., Raharimalala, F. N., Ravelonandro, P., and Mavingui, P. (2013). Diversity of culturable bacteria including Pantoea in wild mosquito Aedes albopictus. BMC Microbiol. 13:70. doi: 10.1186/1471-2180-13-70 
Vestheim, H., Deagle, B. E., and Jarman, S. N. (2011). Application of blocking oligonucleotides to improve signal-to-noise ratio in a PCR. Methods Mol. Biol. 687, 265-274. doi: 10.1007/978-1-60761-944-4_19

Wang, Y., Gilbreath, T. M. III., Kukutla, P., Yan, G., and Xu, J. (2011). Dynamic gut microbiome across life history of the malaria mosquito Anopheles gambiae in Kenya. PLoS ONE 6:e24767. doi: 10.1371/journal.pone.0024767

Wrzosek, L., Miquel, S., Noordine, M. L., Bouet, S., Joncquel Chevalier-Curt, M., Robert, V., et al. (2013). Bacteroides thetaiotaomicron and Faecalibacterium prausnitzii influence the production of mucus glycans and the development of goblet cells in the colonic epithelium of a gnotobiotic model rodent. BMC Biol. 11:61. doi: 10.1186/1741-7007-11-61

Zouache, K., Michelland, R. J., Failloux, A. B., Grundmann, G. L., and Mavingui, P. (2012). Chikungunya virus impacts the diversity of symbiotic bacteria in mosquito vector. Mol. Ecol. 21, 2297-2309. doi: 10.1111/j.1365294X.2012.05526.x

Zouache, K., Raharimalala, F. N., Raquin, V., Tran-Van, V., Raveloson, L. H., Ravelonandro, P. et al. (2011). Bacterial diversity of field-caught mosquitoes, Aedes albopictus and Ae. aegypti, from different geographic regions of Madagascar. FEMS Microbiol. Ecol. 75, 377-389. doi: 10.1111/j.15746941.2010.01012.x

Zouache, K., Voronin, D., Tran-Van, V., Mousson, L., Failloux, A. B., and Mavingui, P. (2009). Persistent Wolbachia and cultivable bacteria infection in the repro- ductive and somatic tissues of the mosquito vector Aedes albopictus. PLoS ONE 4:e6388. doi: 10.1371/journal.pone.0006388

Conflict of Interest Statement: The authors declare that the research was conducted in the absence of any commercial or financial relationships that could be construed as a potential conflict of interest.

Received: 27 January 2014; paper pending published: 13 April 2014; accepted: 21 April 2014; published online: 14 May 2014.

Citation: Minard G, Tran F-H, Dubost A, Tran-Van V, Mavingui P and Valiente Moro C (2014) Pyrosequencing 16S rRNA genes of bacteria associated with wild tiger mosquito Aedes albopictus: a pilot study. Front. Cell. Infect. Microbiol. 4:59. doi: 10.3389/fcimb.2014.00059

This article was submitted to the journal Frontiers in Cellular and Infection Microbiology.

Copyright (C) 2014 Minard, Tran, Dubost, Tran-Van, Mavingui and Valiente Moro. This is an open-access article distributed under the terms of the Creative Commons Attribution License (CC BY). The use, distribution or reproduction in other forums is permitted, provided the original author(s) or licensor are credited and that the original publication in this journal is cited, in accordance with accepted academic practice. No use, distribution or reproduction is permitted which does not comply with these terms. 